\title{
Appendix
}

\section{Overview of the data}

\section{Diplomacy: analysed conferences and meetings}

\section{DipCon $^{1}$}

4th plenary meeting (16 June 1998), U.N. Doc. A/CONF.183/SR.4. 5th plenary meeting (17 June 1998), U.N. Doc. A/CONF.183/SR.5. 6th plenary meeting (17 June 1998), U.N. Doc. A/CONF.183/SR.6. 2nd meeting (16 June 1998), U.N. Doc. A/CONF.183/C.1/SR.2. 3rd meeting (17 June 1998), U.N. Doc. A/CONF.183/C.1/SR.3. 4th meeting (17 June 1998), U.N. Doc. A/CONF.183/C.1/SR.4. 6th meeting (18 June 1998), U.N. Doc. A/CONF.183/C.1/SR.6 7th meeting (18 June 1998), U.N. Doc. A/CONF.183/C.1/SR.7. 8th meeting (19 June 1998), U.N. Doc. A/CONF.183/C.1/SR.8. 9th meeting (22 June 1998), U.N. Doc. A/CONF.183/C.1/SR.9. 10th meeting (22 June 1998), U.N. Doc. A/CONF.183/C.1/SR.10. 26th meeting (8 July 1998), U.N. Doc. A/CONF.183/C.1/SR.26. 29th meeting (9 July 1998), U.N. Doc. A/CONF.183/C.1/SR.29. 32nd meeting (10 July 1998), U.N. Doc. A/CONF.183/C.1/SR.32. 33rd meeting (13 July 1998), U.N. Doc. A/CONF.183/C.1/SR.33. 42nd meeting (17 July 1998), U.N. Doc. A/CONF.183/C.1/SR.42. 9th plenary meeting (17 July 1998), U.N. Doc. A/CONF.183/SR.9.

\section{UN Security Council}

'Civilians in armed conflict' (16 September 1999), U.N. Doc. S/PV.4046.

'International tribunal - Yugoslavia' (20 June 2000), U.N. Doc. S/PV.4174.

'United Nations peacekeeping' (12 June 2003), U.N. Doc. S/PV.4772.

'Liberia' (1 August 2003), U.N. Doc. S/PV.4803.

'Justice and the rule of law: the United Nations role' (24 September 2003), U.N. Doc. S/PV.4833.

'Civilians in armed conflict' (9 December 2003), U.N. Doc. S/PV.4877.

'Complex crises and UN response' (28 May 2004), U.N. Doc. S/PV.4980.

'Civilians in armed conflict' (14 June 2004), U.N. Doc. S/PV.4990.

'Justice and the rule of law: the United Nations role' (6 December 2004), U.N. Doc. S/PV.5052.

'Civilians in armed conflict' (14 December 2004), U.N. Doc. S/PV.5100. 
Appendix

‘Sudan' (31 March 2005), U.N. Doc. S/PV.5158.

\section{UN General Assembly}

(24 September 1997), U.N. Doc. A/52/PV.9.

(8 December 1998), U.N. Doc. A/53/PV.83.

(29 November 1999), U.N. Doc. A/54/PV.64.

(21 November 2000), U.N. Doc. A/55/PV.70.

(13 September 2004), U.N. Doc. A/58/PV.95.

(10 December 2004), U.N. Doc. A/59/PV.70.

UN General Assembly/Sixth Committee (Legal)

(29 October 1996), U.N. Doc. A/C.6/51/SR.26.

(31 October 1996), U.N. Doc. A/C.6/51/SR.28.

(1 November 1996), U.N. Doc. A/C.6/51/SR.29.

(4 November 1996), U.N. Doc. A/C.6/51/SR.31.

(5 November 1996), U.N. Doc. A/C.6/51/SR.32.

(12 November 1996), U.N. Doc. A/C.6/51/SR.38.

(23 October 1997), U.N. Doc. A/C.6/52/SR.14.

(4 November 1998), U.N. Doc. A/C.6/53/SR.9.

(12 December 2000), U.N. Doc. A/C.6/55/SR.9.

(30 November 2004), U.N. Doc. A/C.6/59/SR.27.

\section{International law: considered journals (1998-2005)}

American fournal of International Law (AFIL)

Archiv des Völkerrechts (AVR)

Europäische Grundrechte Zeitschrift (EuGRZ)

European Fournal of International Law (EFIL)

Fournal of International Criminal fustice $(\mathfrak{F I C F})^{2}$

Revue Générale de Droit International Public (RGDIP)

Stanford Fournal of International Law (SFIL)

Zeitschrift für ausländisches öffentliches Recht und Völkerrecht (ZaöRV)

\section{Media: daily newspapers}

Germany (search entry: 'international ${ }^{\star}$ strafgerichtshof ${ }^{\star}$ ')

Frankfurter Allgemeine Zeitung

Frankfurter Rundschau

General-Anzeiger (Bonn)

Hamburger Abendblatt

Stuttgarter Zeitung

Süddeutsche Zeitung

tageszeitung

Welt am Sonntag

United Kingdom (search entry: 'international criminal court')

Evening News (Edinburgh) 
Financial Times

Guardian

Herald (Glasgow)

Independent

Mirror

Observer

The Times

France (search entry: 'cour pénale internationale')

La Croix

Les Echos

Le Figaro

Libération

Le Monde

Sud Ouest

United States (search entry: 'international criminal court')

Arkansas Democrat-Gazette

Baltimore Sun

Buffalo News

Chattanooga Times Free Press

Dayton Daily News

Denver Post

Detroit Free Press

Fresno Bee

Herald Sun

Los Angeles Times

Miami Herald

New York Times

Omaha World Herald

Portland Press Herald

Post-Standard

Rocky Mountain News

San Diego Union-Tribune

San Francisco Chronicle

Salt Lake Tribune

Seattle Post-Intelligencer

St. Louis Post-Dispatch

Tampa Tribune

Tulsa World

USA Today

Washington Post

Washington Times

\section{Data collection periods (media)}

19-26 July 1998 (Adoption of the Rome Statute)

30 June-8 July 2002 (Entry into force of Rome Statute)

12-19 July 2002 (Adoption of Security Council Resolution 1422)

12-19 June 2003 (Adoption of Security Council Resolution 1487) 
15 May-30 June 2004 (Failed renewal of Security Council Resolution 1487)

31 March-6 April 2005 (Adoption of Security Council Resolution 1593)

\section{The legalistic discourse}

a) Discursive arena of diplomacy (in chronological order)

Elizabeth Wilmshurst (UK), UN GA Sixth Committee meeting (31 October 1996), U.N. Doc. A/C.6/51/SR.28, p. 8.

Rolf Welberts (D), UN GA Sixth Committee meeting (1 November 1996), U.N. Doc. A/ C.6/51/SR.29, p. 11.

Hartmut Hillgenberg (D), UN GA Sixth Committee meeting (5 November 1996), U.N. Doc. A/C.6/51/SR.32, p. 10.

Sir Franklin Berman (UK), UN GA Sixth Committee meeting (12 November 1996), U.N. Doc. A/C.6/51/SR.38, p. 6.

Klaus Kinkel (D), UN GA meeting (24 September 1997), U.N. Doc. A/52/PV.9, p. 10.

Rolf Welberts (D), UN GA Sixth Committee meeting (23 October 1997), A/C.6./52/SR.14, p. 9.

Edzard Schmidt-Jortzig (D), DipCon, 4th plenary meeting (16 June 1998), U.N. Doc. A/ CONF.183/SR.4, Vol. II, p. 83.

Bill Richardson (US) DipCon, 5th plenary meeting (17 June 1998), U.N. Doc. A/CONF.183/ SR.5, Vol. II, p. 95.

Sir Franklin Berman (UK), DipCon, 6th plenary meeting (17 June 1998), U.N. Doc. A/ CONF.183/SR.6, Vol. II, p. 98.

Hubert Védrine (F), DipCon, 6th plenary meeting (17 June 1998), U.N. Doc. A/CONF.183/ SR.6, Vol. II, p. 101.

Hans-Peter Kaul (D), DipCon, 3rd meeting (17 June 1998), U.N. Doc. A/CONF.183/C.1/ SR.3, Vol. II, p. 146.

Hans-Peter Kaul (D), DipCon, 7th meeting (18 June 1998), U.N. Doc. A/CONF.183/C.1/ SR.7, Vol. II, p. 184.

Marc Perrin de Brichambaut (F), DipCon, 8th meeting (19 June 1998), U.N. Doc. A/ CONF.183/C.1/SR.8, Vol. II, p. 189.

Hans-Peter Kaul (D), DipCon, 10th meeting (22 June 1998), U.N. Doc. A/CONF.183/C.1/ SR.10, Vol. II, p. 204.

Elizabeth Wilmshurst (UK), DipCon, $2^{\text {th }}$ meeting (9 July 1998), U.N. Doc. A/CONF.183/ C.1/SR.29, Vol. II, p. 295.

Marc Perrin de Brichambaut (F), DipCon, 29th meeting (9 July 1998), U.N. Doc. A/ CONF.183/C.1/SR.29, Vol. II, pp. 295-6.

Hans-Peter Kaul (D), DipCon, 29th meeting (9 July 1998), U.N. Doc. A/CONF.183/C.1/ SR.29, Vol. II, p. 304.

Gerd Westdickenberg (D), DipCon, 33rd meeting (13 July 1998), U.N. Doc. A/CONF.183/ C.1/SR.33, Vol. II, p. 325.

Sir Franklin Berman (UK), DipCon, 33rd meeting (13 July 1998), U.N. Doc. A/CONF.183/ C.1/SR.33, Vol. II, p. 326.

Nancy Soderberg (US), UN GA meeting (8 December 1998), U.N. Doc. A/53/PV.83, p. 12.

Alain Dejammet (F), UN SC meeting: 'Civilians in armed conflict' (16 September 1999), U.N. Doc. S/PV.4046, p. 18.

Sir Jeremy Greenstock (UK), UN SC meeting: 'Civilians in armed conflict' (16 September 1999), U.N. Doc. S/PV.4046, p. 16.

Sir Jeremy Greenstock (UK), UN SC meeting: 'International tribunal - Yugoslavia' (20 
June 2000), U.N. Doc. S/PV.4174, p. 7.

François Alabrune (F), UN GA Sixth Committee meeting (12 December 2000), U.N. Doc. A/C.6/55/SR.9, p. 3.

Michel Duclos (F), UN SC meeting: 'United Nations peacekeeping' (12 June 2003), U.N. Doc. S/PV.4772, p. 24.

Gunter Pleuger (D), UN SC meeting: 'United Nations peacekeeping' (12 June 2003), U.N. Doc. S/PV.4772, p. 25.

Gunter Pleuger (D), UN SC meeting: 'Liberia' (1/08/2003), U.N. Doc. S/PV.4803, p. 4.

Dominique Galouzeau de Villepin (F), UN SC meeting: 'Justice and the rule of law: the United Nations role' (24 September 2003), U.N. Doc. S/PV.4833, pp. 6-7.

Gunter Pleuger (D), UN SC meeting: 'Justice and the rule of law: The United Nations role' (24 September 2003), U.N. Doc. S/PV.4833, pp. 15-16.

Michel Duclos (F), UN SC meeting: 'Civilians in armed conflict' (9 December 2003), U.N. Doc. S/PV.4877, p. 20.

Wolfgang Trautwein (D), UN SC meeting: 'Civilians in armed conflict' (9 December 2003), U.N. Doc. S/PV.4877, p. 25.

Wolfgang Trautwein (D), UN SC meeting: 'Complex crises and UN response' (28 May 2004), U.N. Doc. S/PV.4980, p. 26.

Wolfgang Trautwein (D), UN SC meeting: 'Civilians in armed conflicts' (14 June 2004), U.N. Doc. S/PV.4990, pp. 23-4.

Gunter Pleuger (D), UN SC meeting: 'Justice and the rule of law: the United Nations role' (6 October 2004), U.N. Doc. S/PV.5052, p. 8.

Jean-Marc De La Sablière (F), UN SC meeting: 'Justice and the rule of law: The United Nations role', (6 October 2004), U.N. Doc. S/PV.5052, pp. 20-1.

Jean-Marc De La Sablière (F), UN SC meeting: 'Civilians in armed conflict' (14 December 2004), U.N. Doc. S/PV.5100, pp. 13-14.

Sir Emyr Jones Parry (UK), UN SC meeting: 'Civilians in armed conflict' (14 December 2004), U.N. Doc. S/PV.5100, p. 17.

Gunter Pleuger (D), UN SC meeting: 'Civilians in armed conflict', (14 December 2004), U.N. Doc. S/PV.5100, p. 18.

Jean-Marc De La Sablière (F), UN SC meeting: 'Sudan' (31 March 2005), U.N. Doc. S/ PV.5158, p. 8.

Sir Emyr Jones Parry (UK), UN SC meeting: 'Sudan' (31 March 2005), U.N. Doc. S/PV.5158, p. 7.

\section{b) Discursive arena of international legal experts (in alphabetical order)}

Blanke, Hermann-Josef and Claus Molitor (2001) 'Der Internationale Strafgerichtshof', AVR 39, 142-69.

Carrillo-Salcedo, Juan-Antonio (1999) 'La cour pénale internationale: l'humanité trouve une place dans le droit international', RGDIP 103:1, 23-8.

Cassese, Antonio (1999) 'The statute of the International Criminal Court: some preliminary reflections', EFIL 10:1, 144-71.

Charney, Jonathan I. (1999) 'Progress in international criminal law?', AFIL 93:2, 452-64.

Condorelli, Luigi (1999) 'La Cour pénale internationale. Un pas géant (pourvu qu'il soit accompli...)', RGDIP 103:1, 7-21.

Hafner, Gerhard, Kristen Boon, Anne Rbesame and Jonathan Huston (1999) 'A response to the American view as presented by Ruth Wedgwood', EfIL 10:1, 108-23.

Heselhaus, Sebastian (2002) 'Resolution 1422 (2002) des Sicherheitsrates zur Begrenzung der Tätigkeit des Internationalen Strafgerichtshofs', ZaöRV 62, 907-40. 
Lattanzi, Flavia (1999) 'Competence de la Cour pénale internationale et consentement des états', RGDIP 103:2, 425-44.

Meron, Theodor (1998) 'War crimes law comes of age', AfIL 92:3, 462-8.

Reisman, W. Michael (2004) 'Learning to deal with rejection: The International Criminal Court and the United States', FICF 2:1, 17-18.

Robinson, Darryl (2003) 'Serving the interests of justice: Amnesties, truth commissions and the International Criminal Court', EFIL 14:3, 481-505.

Stahn, Carsten (1998) 'Zwischen Weltfrieden und materieller Gerechtigkeit: Die Gerichtsbarkeit des Ständigen Internationalen Strafgerichtshofs (IntStGH)', EuGRZ 25, 577-91.

Weckel, Philippe (1998) 'La cour pénale internationale. Présentation générale', RGDIP 102:4, 983-93.

Zimmermann, Andreas (1998) 'Die Schaffung eines ständigen Internationalen Strafgerichtshofes. Perspektiven und Probleme vor der Staatenkonferenz in Rom', ZaöRV 58, 47-108.

\section{c) Discursive arena of the media (in chronological order)}

Anthony Lewis, 'U.S. denied its heritage in failing to embrace world court', St. Louis PostDispatch (21 July 1998).

Kenneth Roth, 'Human rights, American wrongs: Europe must resist the US obduracy that is threatening to undermine the International Criminal Court', Financial Times (1 July 2002).

'Justice for all at the ICC', Financial Times (2 July 2002).

'Lamérique, la justice et la paix', Les Echos (2 July 2002).

Paul Meunier, 'La Cour sans les grands', Sud Ouest (2 July 2002).

'For the sake of justice, US should rethink its objection to international court', Herald (2 July 2002).

'Contempt for the law', San Francisco Chronicle (2 July 2002).

Christian Semler, 'Wider die Logik der Erpresser', tageszeitung (5 July 2002).

Erich Rathfelder, 'Bosnien braucht Europa. Die USA gefährden mit ihrem Verhalten den Frieden auf dem Balkan', tageszeitung (5 July 2002).

'Die Souveränität der Staaten bleibt unberührt. Vereinte Nationen. Wahre Gründe des USWiderstands gegen den Internationalen Strafgerichtshof sind unklar', General-Anzeiger (8 July 2002).

Bassir Pour Afsane, 'Le débat à l'ONU sur la Cour pénale internationale confirme l'isolement des Etats-Unis. Un troisième proposition de compromise, présentée par les Americains, maintient l'exigence d'un régime d'exemption général pour leurs GI', Le Monde (12 July 2002).

Franz-Josef Hutsch, 'Recht des Stärkeren statt Stärke des Rechts', Hamburger Abendblatt (15 July 2002).

Stefan Ulrich, 'Der amerikanische Verrat', Süddeutsche Zeitung (15 July 2002).

Andreas Zumach, 'Keine Spur von Europas Stärke', tageszeitung (15 July 2002).

Cokie and Steven Roberts, 'A double standard can bite you back', Tulsa World (16 July 2002).

Terry Olson, 'Une défense de la Cour pénale internationale', Le Figaro (19 July 2002).

Andreas Zumach, 'Erpressung lohnt sich. Internationales Strafgericht: Bundesregierung knickt ein', tageszeitung (14 June 2003).

Steve Crawshaw, 'Why the US needs this court contempt for justice: America's rejection of the International Criminal Court is a threat to its own security', Observer (15 June 
2003).

Clare Short, 'What's next on the horizon for UN?', Evening News (16 June 2003).

William Bourdon, 'La par belle aux bourreaux. La justice pénale internationale est minée par les pays décideurs qui œuvrent pour leur impunité', Libération (11 June 2004).

Steve Crawshaw and Richard Dicker, 'Britain's flawed position on the global court', Financial Times (22 June 2004).

Jean-Christophe Ploquin, 'Soudan. Darfour. Difficile justice', La Croix (1 April 2005).

'Sudan: Saving Darfur', Guardian (1 April 2005).

Bernd Pickert, 'Für Darfur reicht der Fortschritt nicht. Sicherheitsrat bricht US-Widerstand gegen Strafgerichtshof', tageszeitung (1 April 2005).

Matthias Rüb: 'In der Substanz unverändert. Washington läßt dennoch die UN-Resolution zu Darfur passieren', Frankfurter Allgemeine Zeitung (2 April 2005).

Adrian Zielcke, 'Ein Durchbruch. Ahndung von Kriegsverbrechen', Stuttgarter Zeitung (2 April 2005).

'Genocide: Sudan's obvious crime', Seattle Post-Intelligencer (5 April 2005).

'An end to the nightmare', St. Louis Post-Dispatch (5 April 2005).

\section{The interventionist discourse}

a) Discursive arena of diplomacy (in chronological order)

François Legal (F), UN GA Sixth Committee meeting (29 October 1996), U.N. Doc.A/ C.6/51/SR.26, p. 10.

David J. Scheffer (US), UN GA Sixth Committee meeting (31 October 1996), U.N. Doc. A/C.6/51/SR.28, p. 11.

Marc Perrin de Brichambaut (F), UN GA Sixth Committee meeting (4 November 1996), U.N. Doc. A/C.6/51/SR.31, p. 7.

Clive Crook (US), UN GA Sixth Committee meeting (5 November 1996), U.N. Doc. A/ C.6/51/SR.32, p. 4.

Damien Loras (F), UN GA Sixth Committee meeting (23 October 1997), U.N. Doc. A/ C.6/52/SR.14, p. 3.

Bill Richardson (US), UN GA Sixth Committee meeting (23 February 1998), U.N. Doc. A/C.6/52/SR.13, p. 5.

Béatrice le Frapper du Hellen (F), DipCon, 2nd meeting (16 June 1998), U.N. Doc. A/ CONF.183/C.1/SR.2, Vol. II, p. 141.

Bill Richardson (US), DipCon, 5th plenary meeting (17 June 1998), U.N. Doc. A/CONF.183/ SR.5, Vol. II, p. 95.

Hubert Védrine (F), DipCon, 6th plenary meeting (17 June 1998), U.N. Doc. A/CONF.183/ SR.6, Vol. II, p. 101.

François Alabrune (F), DipCon, 6th meeting (18 June 1998), U.N. Doc. A/CONF.183/C.1/ SR.6, Vol. II, p. 177.

Elizabeth Wilmshurst (UK), DipCon, 6th meeting (18 June 1998), U.N. Doc. A/CONF.183/ C.1/SR.6, Vol. II, p. 177.

Marc Perrin de Brichambaut (F), DipCon, 29th meeting (9 July 1998), U.N. Doc. A/ CONF.183/C.1/SR.29, Vol. II, p. 296.

David J. Scheffer (US), DipCon, 29th meeting (9 July 1998), U.N. Doc. A/CONF.183/C.1/ SR.29, Vol. II, p. 297.

David J. Scheffer(US), DipCon, 9th plenary meeting (17 July 1998), U.N. Doc. A/CONF.183/ SR.9, Vol. II, p. 126.

David J. Scheffer (US), UN GA Sixth Committee meeting (4 November 1998), U.N. Doc. 
A/C.6/53/SR.9, p. 8 .

Richard Holbrooke (US), UN SC meeting 'Civilians in armed conflict' (16 September 1999), U.N. Doc. S/PV.4046, pp. 13-14.

David J. Scheffer (US), UN GA Sixth Committee meeting (12 December 2000), U.N. Doc. A/C.6/55/SR.9, p. 5.

James Cunningham (US), UN SC meeting 'United Nations peacekeeping' (12 June 2003), U.N. Doc. S/PV.4772, p. 23.

Gunter Pleuger (D), UN SC meeting 'Liberia' (1 August 2003), U.N. Doc. S/PV.4803, p. 4.

John D. Negroponte (US), UN SC meeting 'Liberia' (1 August 2003), U.N. Doc. S/PV.4803, p. 5.

James Cunningham (US), UN SC meeting 'Justice and the rule of law: The United Nations role' (24 September 2003), U.N. Doc. S/PV.4833, p. 20.

John C. Danforth (US), UN SC meeting 'Justice and the rule of law: The United Nations role' (6 October 2004), U.N. Doc. S/PV.5052, p. 18.

Eric Rosand (US), UN GA Sixth Committee meeting (30 November 2004), U.N. Doc. A/ C.6/59/SR.27, p. 2.

Stuart Holliday (US), UN SC meeting 'Civilians in armed conflict' (14 December 2004), U.N. Doc. S/PV.5100, p. 20.

Anne W. Patterson (US), UN SC meeting 'Sudan' (31 March 2005), U.N. Doc. S/PV. 5158, p. 3.

\section{Additional material (in alphabetical order)}

Richard N. Haass (US State Department Director, Policy Planning Staff), 'Sovereignty: Existing Rights, Evolving Responsibilities', Remarks to the School of Foreign Service and the Mortara Center for International Studies, Georgetown University, Washington, DC (14 January 2003). http://www.state.gov/s/p/ rem/2003/16648.htm (accessed 7 April 2006).

Stewart Patrick (US State Department Policy Planning Staff), 'The Role of the U.S. Government in Humanitarian Intervention', Remarks to the 43rd Annual International Affairs Symposium, 'The Suffering of Strangers: Global Humanitarian Intervention in a Turbulent World', Lewis and Clark College, Portland, Oregon (5 April 2004), http:// www.state.gov/s/p/rem/31299.htm (accessed 7 April 2006).

\section{b) Discursive arena of international legal experts (in alphabetical order)}

Danner, Allison Marston (2003) 'Enhancing the legitimacy and accountability of prosecutorial discretion at the International Criminal Court', AfIL 97:3, 510-52.

Mundis, Daryl A. (2003) 'The Assembly of States Parties and the institutional framework of the International Criminal Court', AFIL 97:1, 132-47.

Scheffer, David J. (1999) 'The United States and the International Criminal Court', AfIL 93:1, 12-22.

Wedgwood, Ruth (1999) 'The International Criminal Court: An American view', EfIL 10:1, 93-107.

\section{c) Discursive arena of the media (in chronological order)}

'A court without the US', Washington Post (21 July 1998).

Fred Hiatt, 'The trouble with the war-crimes court', Washington Post (26 July 1998).

Robert Kagan, 'Europeans courting international disaster', Washington Post (30 June 2002). 
'For the sake of justice, US should rethink its objection to international court', Herald (2 July 2002).

'International Criminal Court. Bush tries shock therapy on U.N.', Herald-Sun (2 July 2002).

'Court politics', The Times (2 July 2002).

Michael Caplan, 'ICC hopes to give peace a chance', The Times (2 July 2002).

'Bush right to reject sway of International Criminal Court. If soldiers commit crimes, their government can handle it', Portland Press Herald (2 July 2002).

'Joining international court would be grave error for U.S.', Tampa Tribune (6 July 2002).

Herbert Kremp, 'Sonderrecht für die USA? Im Streit um den Internationalen Strafgerichtshof will Washington aus gutem Grund nicht einlenken', Welt am Sonntag (7 July 2002).

Michael Nakoryakov, 'U.S. may dislike world court, but staying outside won't help', Salt Lake Tribune (14 July 2002).

Georg F. Will, 'U.S. isn't wary enough of new world court', Seattle Post-Intelligencer (14 July 2002).

'Nach dem Kompromiß', Frankfurter Allgemeine Zeitung (15 July 2002).

Stephen J. Hadley, 'Tribunal is threat to USA', USA Today (15 July 2002).

Ian Buruma, 'Why we must share America's dirty work', Guardian (16 July 2002).

Steve Barrett, 'Don't let foreign court try U.S. troops', Chattanooga Times Free Press (17 June 2003).

'The right to say "no". United States should not be forced to submit to International Criminal Court', Omaha World Herald (23 June 2004).

'The Pentagon and "lawfare", Washington Times (24 June 2005).

A noble compromise', The Times (2 April 2005).

\section{The sovereigntist discourse}

a) Discursive arena of diplomacy (in chronological order)

Damien Loras (F), UN GA Sixth Committee meeting (23 October 1997), U.N. Doc. A/ C.6/52/SR.14, p. 3.

Bill Richardson (US), DipCon, 5th plenary meeting (17 June 1998), U.N. Doc. A/CONF.183/ SR.5, Vol. II, p. 95.

Jamison S. Borek (US), DipCon, 3rd meeting (17 June 1998), U.N. Doc. A/CONF.183/C.1/ SR.3, Vol. II, p. 152.

Hubert Védrine (F), DipCon, 6th plenary meeting (17 June 1998), U.N. Doc. A/CONF.183/ SR.6, Vol. II, p. 101.

David J. Scheffer (US), DipCon, 4th meeting (17 June 1998), U.N. Doc. A/CONF.183/C.1/ SR.4, Vol. II, p. 159.

François Alabrune (F), DipCon, 5th meeting (18 June 1998), U.N. Doc. A/CONF.183/C.1/ SR.5, Vol. II, p. 164.

Marc Perrin de Brichambaut (F), DipCon, 8th meeting (19 June 1998), U.N. Doc. A/ CONF.183/C.1/SR.8, Vol. II, pp. 189-90.

Mr. Scheffer (US), DipCon, 9th meeting (22 June 1998), U.N. Doc. A/CONF.183/C.1/SR.9, Vol. II, p. 195.

Fred Dalton (US), DipCon, 26th meeting (8 July 1998), U.N. Doc. A/CONF.183/C.1/SR.26, Vol. II, p. 280.

Marc Perrin de Brichambaut (F), DipCon, 29th meeting (9 July 1998), U.N. Doc. A/ CONF.183/C.1/SR.29, Vol. II, p. 296.

David J. Scheffer (US), DipCon, 29th meeting (9 July 1998), U.N. Doc.A/CONF.183/C.1/ 
SR.29, Vol. II, p. 297.

David J. Scheffer (US), DipCon, 32nd meeting (10 July 1998), U.N. Doc. A/CONF.183/C.1/ SR.32, Vol. II, p. 322.

Mr. Scheffer(US), DipCon, 42nd meeting (17 July 1998), U.N. Doc. A/CONF.183/C.1/SR.42, Vol. II, p. 361.

David J. Scheffer(US), DipCon, 9th plenary meeting (17 July 1998), U.N. Doc. A/CONF.183/ SR.9, Vol. II, p. 126.

David J. Scheffer (US), UN GA Sixth Committee meeting (4 November 1998), U.N. Doc. A/C.6/53/SR.9, p. 8.

Nancy Soderberg (US), UN GA meeting (8 December 1998), U.N. Doc. A/53/PV.83, p. 11.

François Alabrune (F), UN GA Sixth Committee meeting (12 December 2000), U.N. Doc. A/C.6/55/SR.9, p. 3.

David J. Scheffer (US), UN GA Sixth Committee meeting (12 December 2000), U.N. Doc. A/C.6/55/SR.9, p. 5 .

James Cunningham (US), UN SC meeting 'United Nations peacekeeping' (12 June 2003), U.N. Doc. S/PV.4772, p. 23.

James Cunningham (US), UN SC meeting 'Justice and the rule of law: The United Nations role' (24 September 2003), U.N. Doc. S/PV.4833, pp. 20-1.

Eric Rosand (US), UN GA meeting (13 September 2004), U.N. Doc. A/58/PV.95, p. 5.

John C. Danforth (US), UN SC meeting 'Justice and the rule of law: The United Nations role' (6 October 2004), U.N. Doc. S/PV.5052, p. 18.

Eric Rosand (US), UN GA Sixth Committee meeting (30 November 2004), U.N. Doc. A/ C.6/59/SR.27, p. 2.

Stuart Holliday (US), UN SC meeting 'Civilians in armed conflict' (14 December 2004), U.N. Doc. S/PV.5100, p. 20.

Anne W. Patterson (US), UN SC meeting 'Sudan' (31 March 2005), U.N. Doc. S/PV. 5158, p. 3 .

\section{b) Discursive arena of international legal experts (in alphabetical order)}

Danner, Allison Marston (2003) 'Enhancing the legitimacy and accountability of prosecutorial discretion at the International Criminal Court', AFIL 97:3, 510-52.

Scheffer, David J. (2005) 'Article 98(2) of the Rome Statute: America's original intent', $F I C F$ $3: 2,333-53$.

Scheffer, David J. (2004) 'How to turn the tide using the Rome Statute's temporal jurisdiction', FICF 2:1, 26-34.

Scheffer, David J. (1999) 'The United States and the International Criminal Court', AFIL 93:1, 12-22.

Sur, Serge (1999) 'Vers une cour pénale internationale: la convention de Rome entre les ONG et le Conseil de sécurité', RGDIP 103:1, 29-45.

Wedgwood, Ruth (2005) 'Address to the Cornell International Law Journal Symposium: Milošević and Hussein on Trial', CILF 38:3, 779-87.

Wedgwood, Ruth (1999) 'The International Criminal Court: An American view', EFIL 10:1, 93-107.

\section{c) Discursive arena of the media (in chronological order)}

'A court without the US', Washington Post (21 July 1998).

Fred Hiatt, 'The trouble with the war-crimes court', Washington Post (26 July 1998).

'Bush right to reject sway of International Criminal Court. If soldiers commit crimes, their government can handle it', Portland Press Herald (2 July 2002). 
'Criminal court or rogue court?’, Rocky Mountain News (5 July 2002).

'Joining international court would be grave error for U.S.', Tampa Tribune (6 July 2002).

'Beware of international traps', Chattanooga Times Free Press (8 July 2002).

Georg F. Will, 'U.S. isn't wary enough of new world court', Seattle Post-Intelligencer (14 July 2002).

Stephen J. Hadley, 'Tribunal is threat to USA', USA Today (15 July 2002).

John Rosenthal, 'Les ambiguitiés de la Cour pénale internationale. Bush a raison de s'opposer à la CPI', Le Figaro (16 July 2002).

Steve Barrett, 'U.S. hardly alone in opposing the court', Chattanooga Free Press (16 July 2002).

Steve Barrett, 'Don't let foreign court try U.S. troops', Chattanooga Times Free Press (17 June 2003).

'The right to say "no". United States should not be forced to submit to International Criminal Court', Omaha World Herald (23 June 2004).

\section{The progressivist discourse}

a) Discursive arena of diplomacy (in chronological order)

Revius O. Ortique (US), UN GA meeting (29 November 1999), U.N. Doc. A/54/PV.64, pp. 9-11.

Maurice Halperin (US), UN GA meeting (21 November 2000), U.N. Doc. A/55/PV.70, pp. 11-12.

Yousif Ghafari (US), UN GA meeting (10 December 2004), U.N. Doc. A/59/PV.70, p. 19.

\section{Additional material}

Community of Democracies, 'Final Warsaw Declaration: Toward a Community of Democracies' (27 June 2000), http://www.state.gov/g/drl/rls/26811.htm (accessed 7 April 2006).

Community of Democracies, 'Criteria for Participation and Procedures' (12 September 2000), http://state.gov/g/drl/26085.htm (accessed 7 April 2006).

Community of Democracies, 'Seoul Plan of Action - Democracy: Investing for Peace and Prosperity' (12 November 2002), http://state.gov/g/drl/rls/15259.htm (accessed 7 April 2006).

Paula J. Dobriansky (US State Department Under Secretary for the State of Global Affairs), 'Building Better Democracies and Promoting Democratic Development: The Community of Democracies', Remarks at the Woodrow Wilson International Center for Scholars, Washington, DC (18 October 2002), http://www.state.gov/g/rls/ rm/2002/14516.htm (accessed 7 April 2006).

Paula J. Dobriansky (US State Department Under Secretary for the State of Global Affairs), 'Remarks Delivered by Under Secretary Dobriansky to the Community of Democracies on Behalf of Secretary Powell', Remarks to the Roundtable on Consolidating Democratic Institutions at the Community of Democracies Ministerial Meeting, Seoul, Korea (11 November 2002), http://www.state.gov/g/rls/rm/2002/16254. htm (accessed 7 April 2006).

Kim R. Holmes (US State Department Assistant Secretary for International Organization Affairs), 'Democracy and International Organizations', Remarks to the World Federalist Association and Oxfam, Washington, DC (5 December 2003), URL: http://www.state. gov/p/io/rls/rm/2003/26949.htm (accessed 7 April 2006). 
Appendix

b) Discursive arena of international legal experts

Wedgwood, Ruth (1999) 'The International Criminal Court: An American view', EfIL 10:1, 93-107.

c) Discursive arena of the media (in chronological order)

Trudy Rubin, 'The messy reality of democracy', San Diego Union-Tribune (1 July 2000).

Marc A. Thiessen, 'Prosecution a bad way to remove a dictator. The ability to give dictators a face-saving way out is an essential component of democratic change', Arkansas Democrat-Gazette (16 July 2000).

James Morrison, 'Spirit of democracy', Washington Times (28 July 2000).

\section{Notes}

1 I only analysed those meetings that have been attended by delegates from the four countries under consideration.

2 The first volume of this journal was published in 2003. 